\title{
Effect of nanoSiO2 Additive of Some Mechanical and Water Absorption of Polyvinyl Alchol/Chitosan Blends
}

\author{
Nadia Abbas Ali, Ekram Atta AL-Ajaj, F.T. Mohammed Noori
}

Baghdad University, College Science, Physics Department, IRAQ

\begin{abstract}
Polyvinyl alcohol/Chitosan blend (PVA/CC(50:50) and (PVA/CC/10wt\% $\mathrm{SiO} 2)$ composite, were prepared by casting method on a glass plate. Poly (vinyl alcohol)-chitosan blend films were prepared with a thickness about 250 micron. The crystalline structure of PVA and fingerprint of semicrystalline of chitosan was confirmed by X-ray diffraction. The tensile results show that the tensile strength and Young's modulus of these hybrid films were greatly improved compared to the neat PVA film which was 10.86 $\mathrm{MPa}, 82 \mathrm{GPa}$ respectively and for the sample containing $10 \mathrm{wt} \%$ silica was 20.6 MPa and 227GPa respectively where compared to PVA/CC which was $15.93 \mathrm{MPa}$ and 131GPa respectively. Strong interfacial bonding between the silica and the PVA/CC, and homogenous distribution of the silica particles in PVA/CC are supportive of markedly improved mechanical strength. The solubility measurements showed that the hybrid has an enhanced water resistance, the solubility decreased with the addition of silica. Blending PVA and chitosan improved strength and young modules of the film and increased water uptake because hydrophobicity of two polymers blend films.
\end{abstract}

Key words: PVA, Chitosan , Nano SiO2 XRD,FTIR, Tensile strength, Water Absorption

\section{INTRODUCTION}

There is growing interest in recent times to develop materials with film forming capacity and having antimicrobial properties which improve food safety and shelf-life. Antimicrobial packaging is one of the most promising active packaging systems, the use of such packaging is not meant to be a substitute for goods animation practices, but it should enhance the safety of food as an additional hurdle for the growth of pathogenic and/or spoilage microorganisms(Tripathi etal, 2009). Barrier film technologies protect food from deterioration by reducing or eliminating oxygen and water vapor permeation. Barrier films are becoming more widespread, in part because of increasing popularity of prepackaged food (EL-HEFIAN etal, 2010).

Poly(vinyl alcohol) (PVA) a biodegradable, synthetic polymer, innocuous, non-carcinogenic and have good biocompatible properties. Poly (vinyl alcohol) has excellent film forming properties. Because of its good film forming and highly hydrophilic water soluble with 
outstanding chemical stability it was blended different synthetic and natural polymers, the use of this polymer is important in many applications such as controlled drug delivery systems, membrane preparation, recycling of polymers and packaging (Duncan, 2011). Chitosan also represents interesting properties such as excellent film forming capacity and gas and aroma barrier properties at dry conditions, which makes it a suitable material for designing food coatings and packaging structures. All the above-mentioned properties also make chitosan a very good candidate to design novel antimicrobial active packaging technologies to improve the quality and safety and to extend the shelf-life of perishable foods (Zheng etal, 2001).

A number of other nanomaterials can be added on their own, or in addition to nanoclays, to polymers to provide additional barrier or functional properties for food packaging purposes. These include metal and metal oxide nanoparticles, nanofibres and nanotubes. Silica nanoparticles can be inserted into certain polymer matrices (such as polypropylene or starch) to improve mechanical or barrier properties of composites and biodegradable films. The purpose of this work is to study the optical, physical, and tensile properties of PVA/Cs blend with addition of nano silica dioxide $\mathrm{SiO} 2$ composite.

\section{EXPERIMENTAL WORK}

\section{Materials}

PVA was purchased from Aldrich Co. (with99\% hydrolyzed, Mw 16,000) chitosan (CC) (high M.W, > 75\% deacetylated) is purchased from Sigma Chemical Company (St. Louis, USA).Acetic acid (glacial, 99-100\%), distilled water is used for the preparation , nanoSiO2 partical size $(20 \mathrm{~nm})$ from sima-aldrch company

\section{Preparation of PVA/Chitosan blend Films}

$50 \mathrm{ml}$ of chitosan solution (1\% wt./wt. in acetic acid) and50 $\mathrm{ml}$ of PVA solution (1\% wt./wt. in water) (1:1) are mixed in $250 \mathrm{ml}$ beaker and stirred for $1 \mathrm{~h}$ at $60 \mathrm{oC}$ to obtain homogeneous solution. The solution is transferred immediately into polystyrene Petri dishes and dried at $60 \mathrm{oC}$ in an electric oven for $48 \mathrm{~h}$. The films were peeled off and measured thickness in micrometer found it is $250 \mu \mathrm{m}$ a and kept under evacuated desiccators over fresh silica gel until use.

\section{Preparation of PVA/CC /nanoSiO2 Films}

PVA/CC nanocomposites preparation in weight $(10 \mathrm{wt} \%)$ the nanoSiO 2 are weighted and manually mixed with PVA/CC blend were mixed by shearing mixer at $800 \mathrm{rpm}$ for 15 minutes to have good distribution .Distribution of the PVA/CC/SiO2 components on the surface of these composites was investigated by optical microscopy, was compared with that in the PVA/CC and appear good distribution $\operatorname{Fig}(2 \mathrm{a}, \mathrm{b})$ 


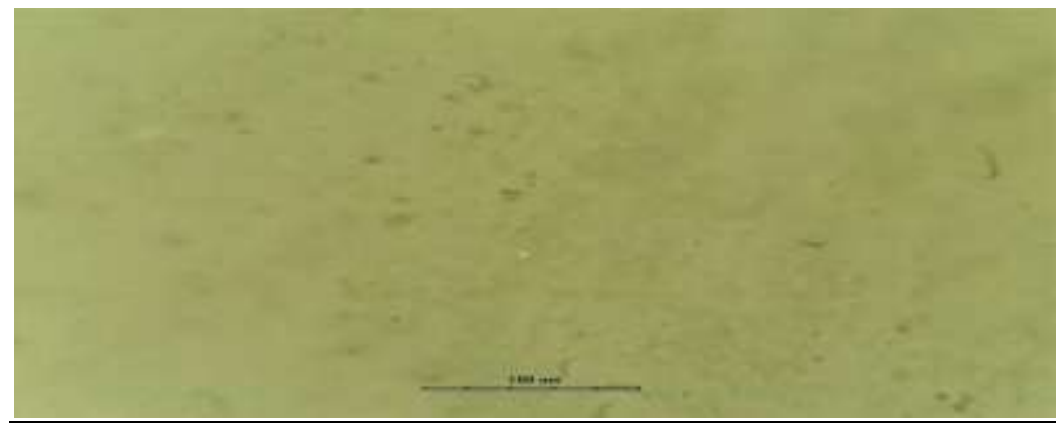

b: -PVA/CC/SiO2

Fig(1): Optical microscopy of blend and composites PVA

\section{FTIR}

Spectra were recorded in $\mathrm{KBr}$ discs on a Shimadzu FTIR model 8000 Testcan IRspectrometer under dry air at room temperature within the wave number range of $4,000-$ $500 \mathrm{~cm}-1$.

\section{X-ray Diffraction}

X-ray Diffraction was done using a Brüker D8 Advance instrument, at $40 \mathrm{KV}, 40 \mathrm{~mA}$ using target $\mathrm{Cu} \mathrm{K \alpha}$ with secondary monochromator (Karlsruhe, Germany)

\section{Tensile Properties}

The samples were prepared as films and their tensile strength using (Instron Co., Norwood, MA, USA), with a $65 \mathrm{~N}$ load cell). Was used to measure tensile strength, young's modulus .The tests were carried out according to ASTM D-638 standard. Two metallic grips were attached for griping both ends of the test specimen of the film. The modulus of elasticity (Young's modulus) was determined according to the following equation

Tensile strength $\left(\mathrm{N} / \mathrm{mm}^{2}\right)=$ Force $(\mathrm{F}) /$ Area $(\mathrm{A}) \ldots \ldots \ldots \ldots \ldots . . .1$

Elongation $(\%)=$ Increase in length $(\Delta \mathrm{L}) /$ Original length $\left(\mathrm{L}_{0}\right) \times 100 \% \ldots . . . . . . . . . . .2$

Young modulus $=\mathrm{F}$ Lo/A $\Delta \mathrm{L} \ldots \ldots . . . \ldots \ldots . . .3$

Where:

F: force exerted on an object under tension, $\mathrm{L}_{0}$ : original length, $\mathrm{A}$ :cross section area, $\Delta \mathrm{L}$ :

length of the object changes

\section{Water Uptake}

The water uptake of different samples was calculated using the following method:

Water uptake $(\mathrm{M} \%)=100 \times\left(\mathrm{W}_{2}-\mathrm{W}_{1}\right) / \mathrm{W}_{1}$ .4

Diffusion Coefficient $(\mathrm{D})=\pi(\mathrm{Kb} / 4 \mathrm{M} \infty)^{\wedge^{2}} \ldots \ldots \ldots \ldots . . .5$

Permeability $(\mathrm{P})=1000(\Delta \mathrm{M}) / \mathrm{NV} \ldots \ldots \ldots \ldots \ldots \ldots . \ldots 6$

Where:

$\mathrm{W}_{1:}$ is the weight of completely dried sample, and $\mathrm{W}_{2}$ is the weight of swelled sample in solution at $25 \mathrm{C}$ for 7 days , $\mathrm{K}$ : is the slope of curve between mass gain and immersion time , b: thickness of sample , $\mathrm{M} \infty$ :saturation moisture mass , $\mathrm{N}$ number of day stored sample , $\mathrm{V}$ : volume of container cup. 


\section{RESULTS AND Discussion}

\section{FTIR}

FTIR spectra of chitosan andPVA/CC film are shown in Fig(3) Stretching vibration spectra of the amide group of chitosan films appear at $1560 \mathrm{~cm}-1$. The change in the characteristic shape of the chitosan spectrum as well as shifting of peak to a lower frequency range due to hydrogen bonding between - $\mathrm{OH}$ of PVA and $-\mathrm{OH}$ or $-\mathrm{NH} 2$ of chitosan in the blended films. This is due to the fact that when two or more polymers are mixed, changesin characteristic spectra peaks occur due to the reflection of the physical blends and chemical interactions. These observations indicate the existence of good miscibility between chitosan and PVA(50:50) and this is most likely due to the formation of intermolecular hydrogen bonds between the amino and hydroxyl groups in chitosan and the hydroxyl groups in PVA(Parvin etal, 2010).

The results obtained in this study indicated that the nano- $\mathrm{SiO}_{2}$ particles were dispersed evenly within the chitosan /PVA and an intermolecular hydrogen bond and a strong chemical bond $\mathrm{C}$ $-\mathrm{O}=\mathrm{Si}$ were formed in the nano- $\mathrm{SiO}_{2}$ and chitosan /PVA. A hydrogen bond was formed in nano- $\mathrm{SiO}_{2}$ and chitosan / polyvinyl alcohol (PVA), and intermolecular hydrogen bonding of the chitosan was decreased at the addition of nano-SiO2. Meanwhile, the chemical bond C-O$\mathrm{Si}$ was also formed in nano-SiO $2 /$ chitosan /PVA hybrid materials; therefore, the miscibility and compatibility between chitosan and PVA were increased (Silva etal, 2013).

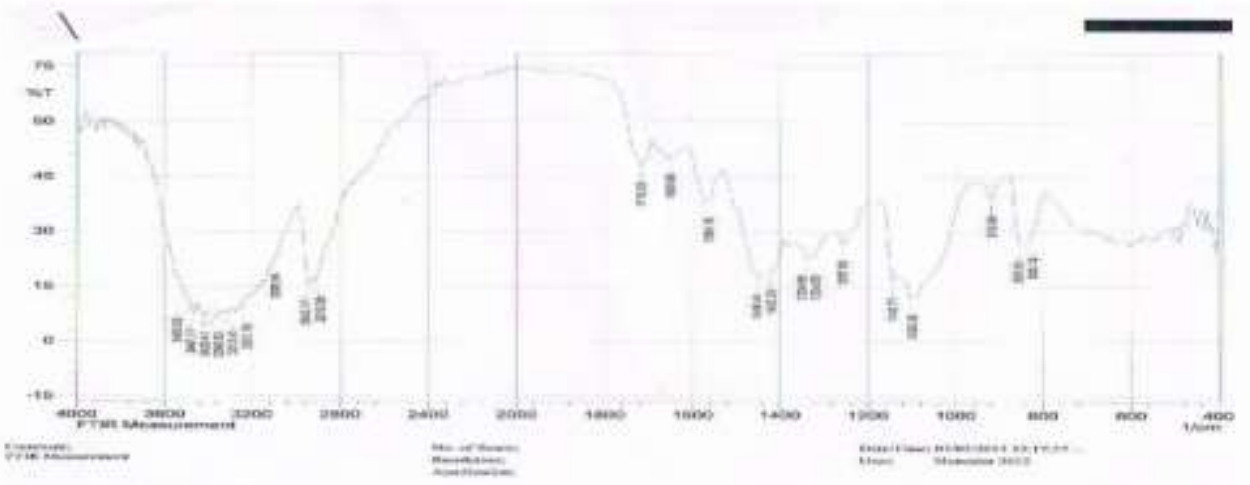

a

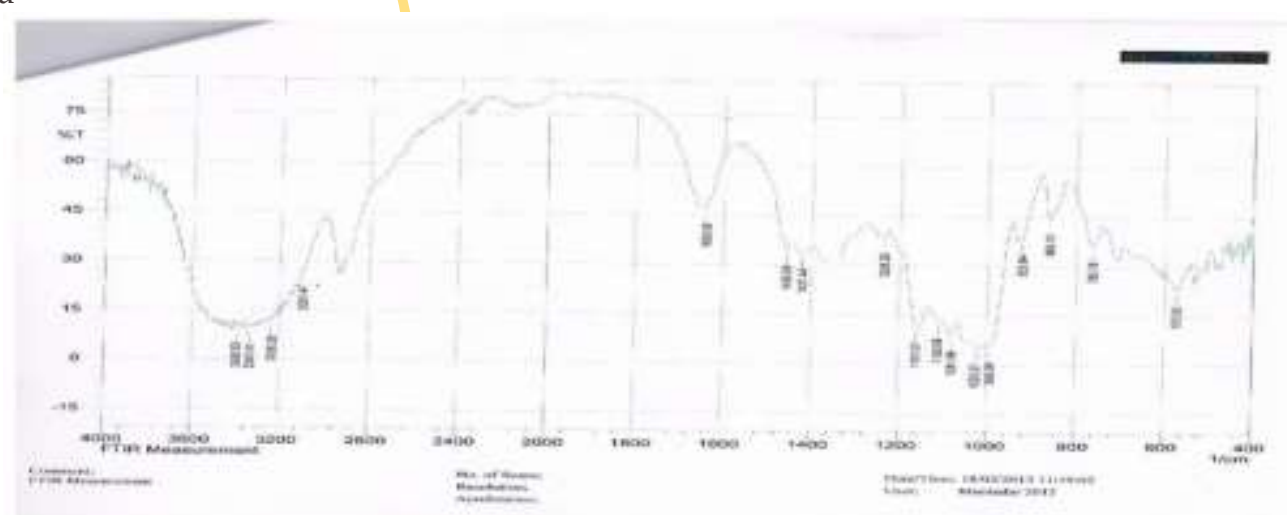

b 


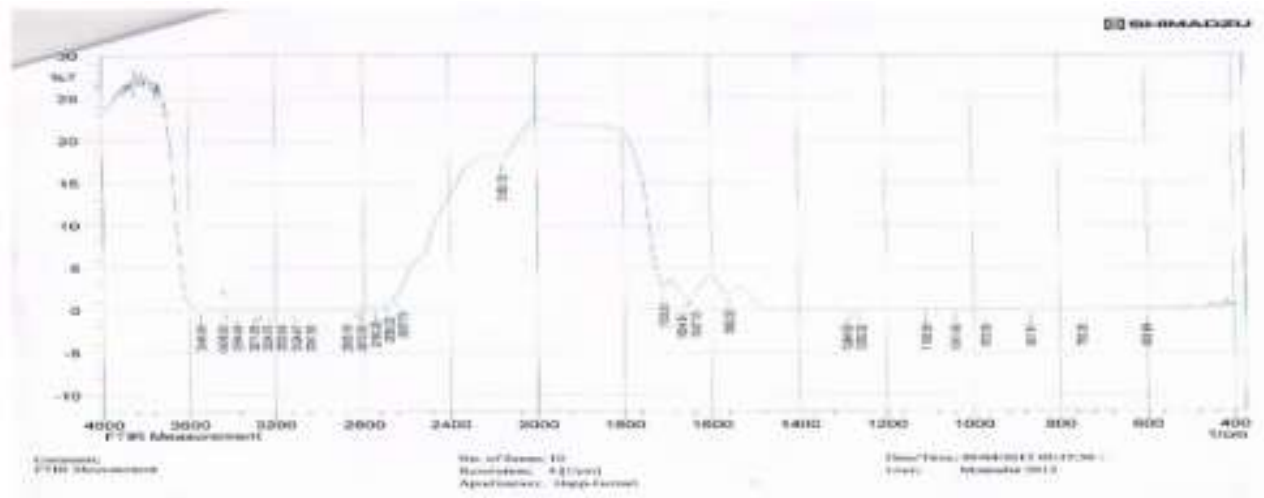

C

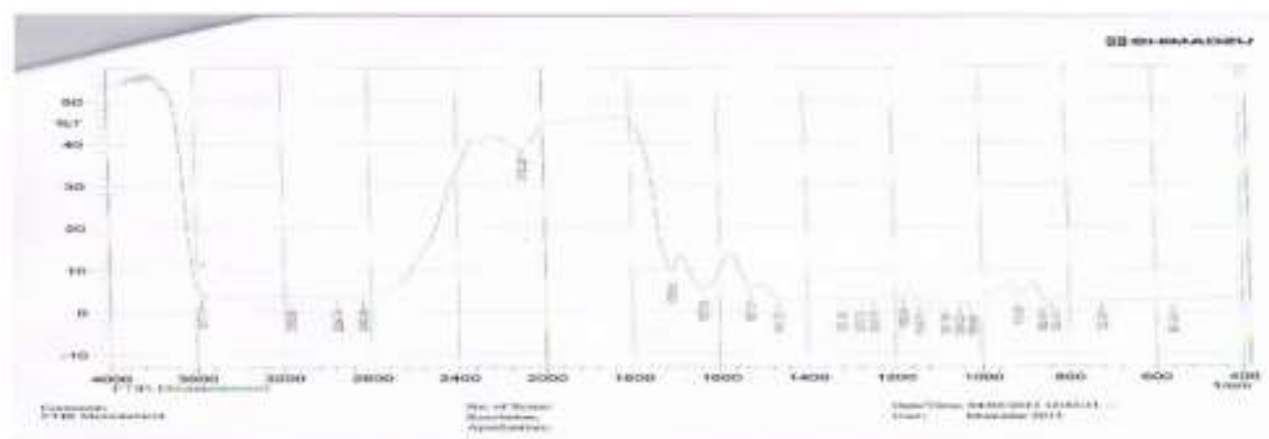

d

Fig(2):FTIR of a:PVA, b:chitosan, $c:$ PVA/CC, d:PVA/CC/SiO2

\section{X-ray}

X-ray diffraction is a proven tool to study crystal lattice arrangements and yields very useful information on degree of sample crystallinity. X-ray patterns of polyvinyl alchol is located at $2 \theta=16^{\circ}$ and $23^{\circ}$ and they are strong and intense indicating the high crystalline structureof PVA. The XRD pattern of chitosan exhibits broad diffraction peaks at $2 \theta=$ $10^{\circ}$ and $20^{\circ}$ which are typical fingerprints of semi-crystalline. Md. Monarul et.al have find that x-ray of chitosan exhibits broad diffraction peaks at $2 \theta=10^{\circ}$ and $21^{\circ}$ which are typical fingerprints of semi-crystalline chitosan (Islam etal, 2008).

X-ray diffraction patterns of chitosan: $\operatorname{PVA}(50: 50)$ show diffractive peak in the region from $2 \theta=20$.

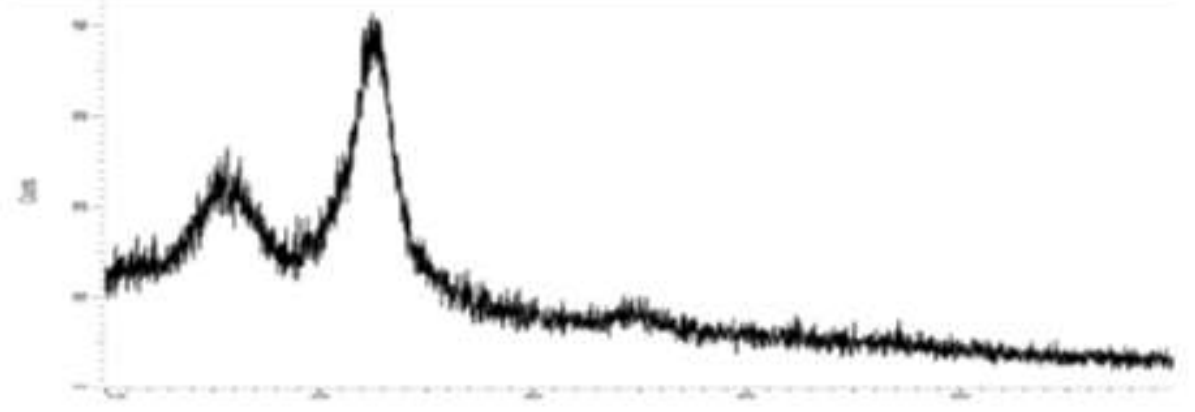




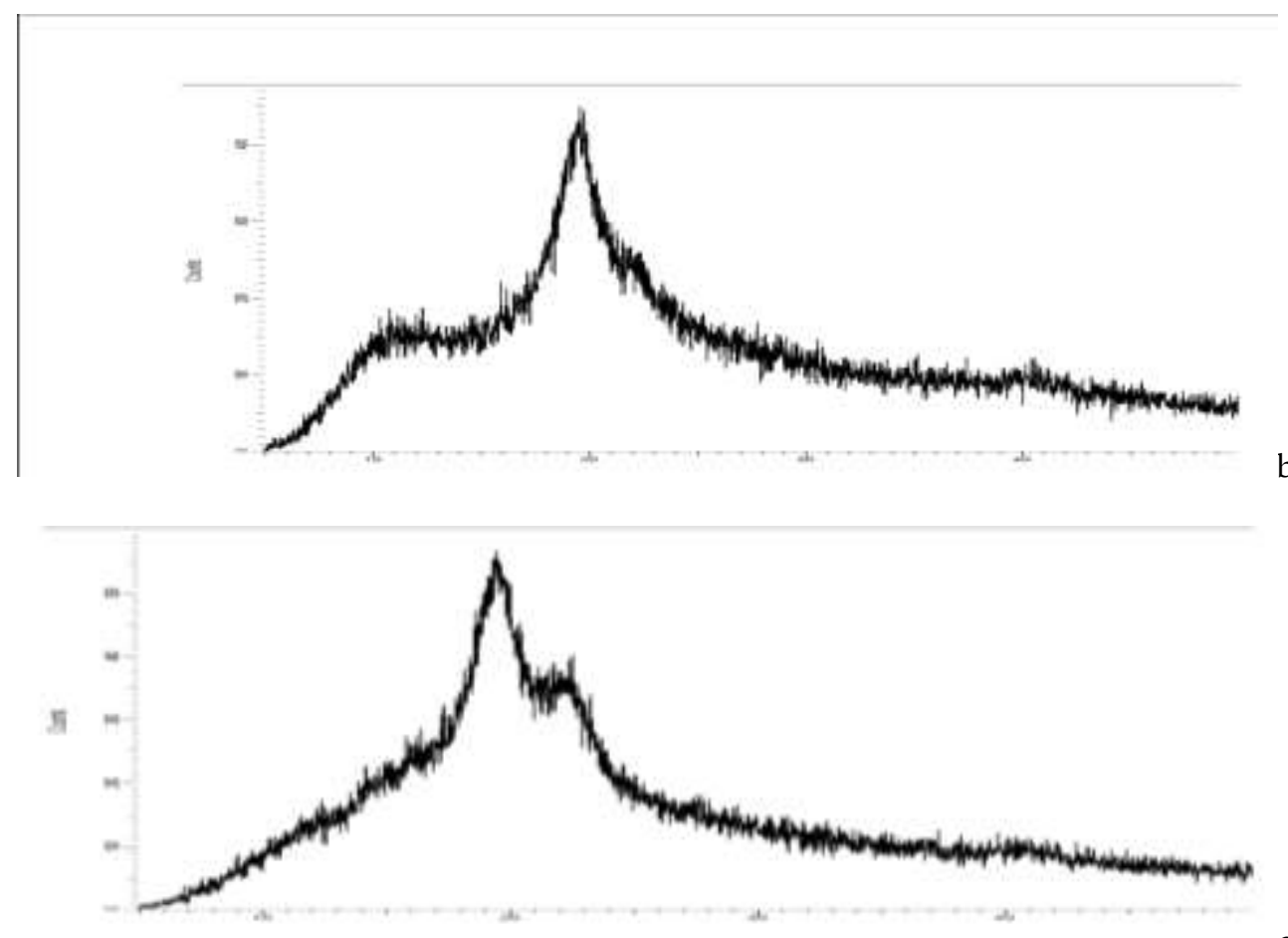

b

Fig(3) X-ray diffraction spectra of a: PVA b:chitosan c:PVA/CC blend

\section{Tensile Properties}

The tensile testing provides an indication of the strength and elasticity of the films, which can be reflected by strength and strain-at-break. The pure PVA film showed the tensile strength at 10.86 MPa. Blending PVA-CC improved tensile strength compared of PVA and become 15.93, . These results indicate that blend films have higher tensile strength than pure PVA films. S. Bahram et.at have also supported this results and find of blend PVA/CC that tensile strength is $19 \mathrm{MPa}($ Bahrami etal, 2003). When add nano $\mathrm{SiO} 2(10 \%)$ the tensile strength and Young modulus increased and become $20.6 \mathrm{MPa}$ and 227GPa respectively that because nanoparticles have significant surface effects, size effects, that improve material properties.

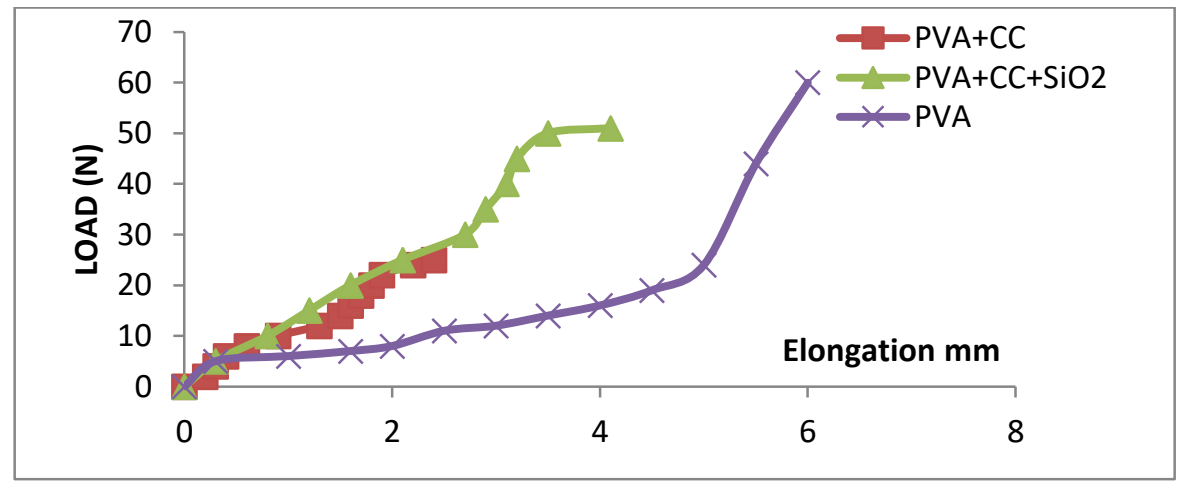

Fig(4) load and elongation curves of PVA, PVA/CC, PVA/CC/SiO2 
Table (1) Mechanical properties of blended and composites films

\begin{tabular}{|c|c|c|}
\hline Sample & Tensile strength(MPa) & Young modulus (GPa) \\
\hline PVA & 10.86 & 82 \\
\hline PVA/CC 50:50 & 15.93 & 131 \\
\hline PVA/CC/SiO2(10\%) & 20.6 & 227 \\
\hline
\end{tabular}

\section{Water Uptake and permeability}

As shown in Table (2) water uptake of pure PVA decrease because nature of hydrophilic and depends upon only hydroxyl groups $(\mathrm{OH})$ and blend of chitosan is increased the hydrophilicity because its hydroxyl groups $(\mathrm{OH})$ and amino groups (NH2) while that of PVA depends upononly hydroxyl groups $(\mathrm{OH})$. Therefore hydroxyl group can form stronger hydrogen bonds with water $(\mathrm{H} 2 \mathrm{O})$ than an amino group. This could be one reason why membranes with a higher content of chitosan (50-100 wt.\%)in the polymer produced higher water permeation fluxes (Svang-Ariyaskul etal, 2008). The water uptake of the composites are lower than the PVA/CC blend because contain nano $\mathrm{SiO} 2$ decreases nano particle are spread throughout the plastic and are able to block oxygen, carbon dioxide and moisture from reaching fresh meats or other foods(Shao etal, 2011).

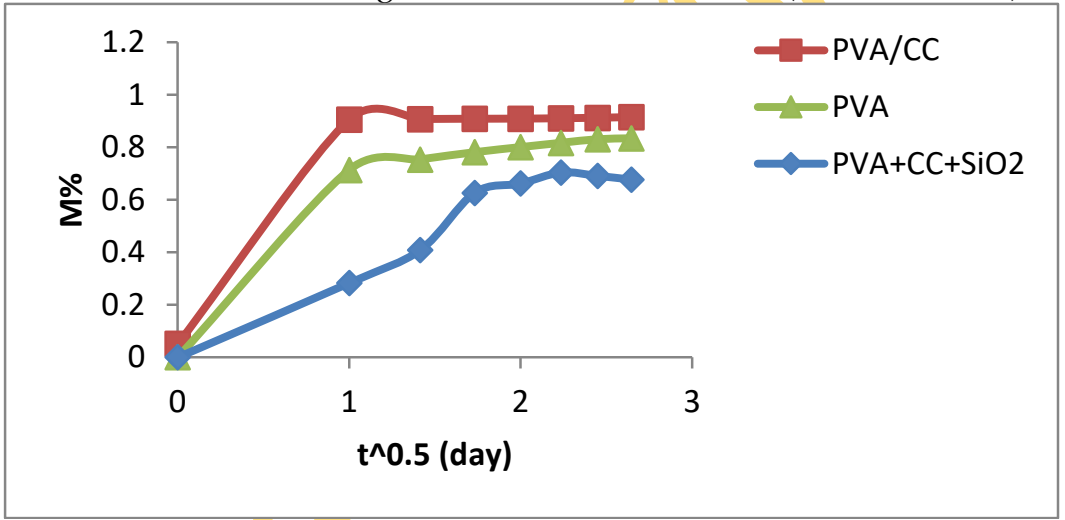

Fig(5): Water Uptake of blend and composites films .

Table (2): Water Uptake and permeability of blended and composites films.

\begin{tabular}{|l|l|l|}
\hline Sample & $\mathbf{D}\left(\mathbf{m m}^{2} /\right.$ day $)$ & P(gm/day.ml) \\
\hline PVA pure & $2.77 \mathrm{E}-07$ & 4.385714 \\
\hline PVA/CC 50:50 & $3.69 \mathrm{E}-07$ & 5.785714 \\
\hline PVA/CC/SiO2(10\%) & $1.54 \mathrm{E}-05$ & 3.407143 \\
\hline
\end{tabular}

\section{CONCLUSION}

In this study the blending of PVA /CC film prepare under $70 \mathrm{C}^{\circ}$ and composites in presence of nano $\mathrm{SiO}_{2}$, have been successfully prepared by the solution-casting technique. The FTIR XRD give the characteristic of pure PVA, Chitosan , PVA/CC, PVA/CC/SiO2, tensile strength an Young modulus of composites increased compare in PVA pure and blend of chitosan. 


\section{REFERENCE}

A. Svang-Ariyaskul, R.Y.M. Huang, P.L. Douglas, R. Pal, X. Feng, P. Chen, L. Liu" Blended chitosan and polyvinyl alcohol membranes for the pervaporation dehydration of isopropanol" Journal of Membrane Science 280 , 815-823,2008.

ESAM A. EL-HEFIAN*, MOHAMED MAHMOUD NASEF§ andABDUL HAMID YAHAYA"The Preparation and Characterization of Chitosan /Poly (Vinyl Alcohol) Blended Films" E-Journal of Chemistryt 7(4), 1212-1219, 2010,

Fábio E. F. Silva, Maria Carolina B. Di-Medeiros, Karla A. Batista, andKátia F. Fernandes PVA/Polysaccharides Blended Films: Mechanical Properties Journal of Materials 2013

Fahmida Parvin, Md. Arifur Rahman, Jahid M. M. Islam, Dr. Mubarak A. Khan, A. H. M.Saadat, Preparation and Characterization of Starch/PVA Blend for biodegradable Packaging Material, Advanced Materials Research Vols. 123-125 , pp 351-35 , 2010

Hua Zheng, Yumin Du ${ }^{*}$, Jiahui Yu,Ronghua Huang, Lina Zhang ${ }^{2}$ "Preparation and characterization of chitosan/poly(vinyl alcohol) blend fibers", Journal of Applied Polymer

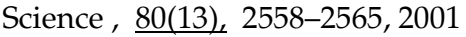

Lu Shao*, Chenzhong Mu, Yifeng Huang, Shangang Ding, Xiao Li,Yongping Bai, and Yudong Huang," Enhancement of Ink-receiving Properties ofSiO2/PVA Composite films by UsingRare Earth-modified SiO2 Particles", Iranian Polymer Journal, 20 (12), 955-967, 2011,.

Md. Monarul Islam, Shah Md. Masumb, M. Mahbubur Rahmana, Md. Ashraful Islam Mollab, A. A. Shaikhc, S.K. Roya "Preparation of Chitosan from Shrimp Shell and Investigation of Its Properties"International Journal of Basic \& Applied Sciences IJBAS-IJENS 11 ( 77), 2008.

S. Bahram Bahrami, Soheila, S. Kordestani1, Hamid Mirzadeh1and Parvin Mansoori" Poly (vinyl alcohol) - Chitosan Blends Preparation, Mechanical and Physical Properties" Iranian Polymer Journal, 12 (2), 139-146,2003.

S. Tripathi, G.K. Mehrotra, P.K. Dutta," Physicochemical and bioactivity of cross-linked chitosan-PVA film for food packaging applications" , International Journal of Biological Macromolecules 45 , 372-376,2009.

Timothy V. Duncan" Applications of nanotechnology in food packaging and food safety: Barrier materials, antimicrobials and sensors", Journal of Colloid and Interface Science, Sci., 7(17), (2011) 